\title{
The Road of Urban-Rural Integration Development Under the Rural Revitalization Strategy
}

\author{
Ma Xiaoshuang \\ Academy of Marxism, Jiangsu University, Zhenjiang, China \\ Email address: \\ 915552886@qq.com \\ To cite this article: \\ Ma Xiaoshuang. The Road of Urban-Rural Integration Development Under the Rural Revitalization Strategy. International Journal of \\ Agricultural Economics. Vol. 4, No. 4, 2019, pp. 181-185. doi: 10.11648/j.ijae.20190404.16
}

Received: May 13, 2019; Accepted: July 1, 2019; Published: July 12, 2019

\begin{abstract}
In the process of promoting rural revitalization, the western developed countries focus on legislation, that is, formulating relevant laws and regulations, strengthening rural infrastructure construction, scientifically and rationally formulating rural regional planning plans, implementing special support plans for rural areas, and vigorously developing rural human resources to give full play to the leading and supporting role of science and technology. Drawing on the experience of developed countries and starting from China's national conditions, the 2018 No.1 central document sets out detailed requirements and arrangements for the rural revitalization, this strategy is a major decision to promote urban-rural integration development and the only way to build a moderately prosperous society in all respects. This strategy captures and solves the outstanding problems that restrict the development of agriculture and rural areas, improves the scientific and rational guarantee mechanism, strengthens the construction of rural infrastructure, promotes the integration of urban and rural areas, vigorously develops rural enterprises, promotes the integration and development of rural industries, and provides a strategy for rural revitalization, support the public sector and improve the rural governance structure to promote the integration of urban and rural areas. On the basis of combing the strategy of rural revitalization and the theory of urban-rural integration, this paper deeply explores the development constraints of urban-rural integration under the background of the Rural Revitalization Strategy, and puts forward the corresponding solutions.
\end{abstract}

Keywords: Rural Revitalization, Urban-rural Integration, Path

\section{Introduction}

In China, now the development of rural areas is closely related to the development of the whole society and people's satisfaction for a better life. The Rural Revitalization Strategy and the Regional Coordinated Development Strategy fully demonstrate the important position of the issues of agriculture, rural areas and rural residents in China's socialist modernization and the determination of the CPC central committee to solve the problem of the uncoordinated development of rural areas. The Rural Revitalization Strategy involves the construction of rural economy, politics, culture and other aspects, rural-urban integrated development is based on revitalization, so it is the only way to promote rural-urban integrated development with the strategy of rural revitalization. [1]

\section{The Basic Connotation of the Rural Revitalization Strategy}

The report of the 19th CPC national congress gives a comprehensive overview of the basic connotation of the Rural Revitalization Strategy, pointing out "We should give priority to the development of agriculture and rural areas, establish and improve the system, mechanism and policy system for rural-urban integrated development, and accelerate the modernization of agriculture and rural areas in line with the general requirements of industries prosperity, ecological livability, civilized local customs, effective governance and a prosperous life." [2] 


\subsection{Take the Priority to Agricultural and Rural Development as the Basic Premise}

Economic revitalization is the key point of rural revitalization, and it is also the latest and most fundamental problem of Rural Revitalization Strategy. To promote rural economic development, we must vigorously develop rural industries, and rural industry should be based on agriculture, therefore, it is necessary to adhere to the path of modern agriculture and realize the synchronous development of new industrialization, informatization, urbanization and agricultural modernization. China is in the decisive period of building a moderately prosperous society in an all-round way, puts forward more urgent requirement to promote agricultural modernization development. Therefore, it is a key task in the new era to give priority to rural regional development and agricultural modernization and balance urban and rural development.

\subsection{Take Industries Prosperity, Ecological Livability, Civilized Local Customs, Effective Governance and a Prosperous Life as the General Requirements of the Strategy}

Industries prosperity is the key point of rural revitalization. Economic revitalization is the key and fundamental point of rural revitalization, it takes industrial revitalization as guarantee. Therefore, the primary task of Rural Revitalization Strategy is to promote industrial revitalization. Ecological livability is the premise of village revitalization strategy. The fundamental purpose of rural revitalization is to improve the quality of life and the level of rural residents. It is not a simple economic revitalization, but the coordinated development of economy, politics, culture and other aspects. Rural revitalization not only needs material support, but also needs spiritual support. On the basis of meeting the material conditions, enriching the spiritual and cultural life of the rural residents will meet the requirements of a better life. Effective governance is the guarantee of rural revitalization. Scientific governance, democratic governance is the fundamental way that achieves effective governance. Prosperous life is the foundation of rural revitalization. The fundamental purpose of rural revitalization is to improve the quality of life and the standard of living of rural residents. Prosperous life is the guarantee for rural residents to live better, but also to the basic standard to test the Rural Revitalization Strategy.

\subsection{Take Establishment and Improvement of the System, Mechanism and Policy System for Integrated Urban and Rural Development as the Key Link}

Since the reform and opening up 40 years ago, China's urbanization process has accelerated, and the status quo of the urban-rural Dual Structure has not been solved, on the contrary, the imbalance between urban and rural development is increasingly prominent. [3] In order to realize the integrated development of urban and rural areas, the dual structure of urban and rural areas must be solved first, otherwise the strategy of rural revitalization will lose its own significance. Therefore, the top-level design of the Rural Revitalization Strategy should be accelerated to provide an inexhaustible driving force for the Rural Revitalization Strategy in terms of the system and mechanism of urban-rural integrated development.

\subsection{Take the Acceleration of Agricultural and Rural Modernization as the Fundamental Goal}

Agricultural modernization is the weakest link in building a moderately prosperous society in all respects in China. The present situation of large rural population and unbalanced and inadequate development in China indicates the opportunity and potential of future development on the other hand. The rapid development of industrialization and urbanization in China has laid a solid foundation for the development of rural areas and provided the foundation and conditions for industry and cities to nurture rural areas. Therefore, it is necessary and feasible to accelerate the modernization of agriculture and countryside and take the rural revitalization as the goal.

\section{Difficulties Faced by China's Rural Development}

The imbalance of urban and rural development in China is becoming more and more serious, which hinders the process of urban and rural integration to some extent. Too much emphasis on urban development leads to the lag of rural development, which has a great impact on the balanced development of the whole society.

\subsection{Rural Hollowing, Aging Phenomenon Is Serious, Lack of Relevant Supporting Mechanism}

Rural construction needs a complete and predictable development plan to ensure the coordinated, unified and sustainable development of rural areas. At present, there are many undesirable phenomena in rural areas of China, such as unchecked construction and waste of resources caused by the abuse of land resources, etc., which further damage the ecological environment when the economy does not get developed. Agricultural production cannot be modernized and scaled, and the production and life styles of many poor areas are still backward, and the life happiness of rural residents cannot be effectively improved.

Rural development needs corresponding supporting mechanism, which is not only the guarantee of rural development, but also the booster for the coordinated development of rural areas. The advancement of urbanization has led to increasingly prominent problems in land, household registration and talent development. Due to the restrictions of the household registration system, many rural migrant workers entering big cities cannot truly enjoy the public services of urbanization similar to that of urban residents. For rural migrant workers, "the cities they can't integrate into and the villages they can't go back to" greatly reduces their happiness index, affecting the harmonious development of the whole society. [4] 


\subsection{Rural Migrants Do Not Have Equal Access to Public} Services

Currently, the development of urbanization in China results in some resources flowing to cities and towns in a single direction for the support of urban economic development, which makes the elements needed to support agricultural production and rural economic development miss. A large number of young people go to cities, lots of farmland are desolated, rural industries shrink, due to the lack of population and the backward production and lifestyle in some poor rural areas, the rural situation decays, seriously affecting the development of China's modernization.

Rural migrant workers, as urban builders, have no urban household registration and cannot enjoy the benefits and treatment they deserve, finally they have to go back to rural areas for development. Every year in China, a large number of farmers who work in cities and towns do household registration reform, so that they can take root in cities and become "city residents", enjoy their due benefits and treatment to improve the index of residents' happy life. [5] Reducing the rural population by integrating more rural population into the cities and towns is conducive to the industrialization and large-scale operation of rural agriculture and accelerating the development of rural areas.

\subsection{Ecological Environmental Pollution Is Serious, and the Relationship Between Economic Development and Ecological Environmental Protection Is Unbalanced}

What the rural development lacks is the environmental protection mechanism. For a long time, the rural environmental protection lacks mechanism, and its environmental prevention and control cannot get the balanced treatment with the urban areas, which will deviate from the current situation of the people's growing need for a better life. [6] For a long time, film and other wastes in agricultural production cannot be properly disposed, causing serious damage to the land and soil. The straw produced in crops is only used for incineration, which brings a certain degree of pollution to the atmosphere; secondly, farmers' weak awareness of environmental protection leads to the phenomenon of garbage discarded everywhere; the excessive use of pesticides causes quality problems of agricultural products, which seriously affects the physical and mental health of residents. To maintain the sustainable development of our society, we need to focus on the protection of human rights and create a harmonious, green, orderly and healthy ecological environment.

\section{The Choice of the Path of Urban-Rural Integrated Development Under the Rural Revitalization Strategy}

The release of the 2018 No.1 central document is the top-level design of the revitalization strategy for rural areas in the new era, highlighting the important role of agriculture, rural areas, and rural residents issues in the process of China's socialist modernization. Further establishment and improvement of relevant systems and mechanisms for coordinated development between urban and rural areas and between regions and realization of integrated development between urban and rural areas is an important approach for China's rural development in the new era. [7]

\subsection{Link with the Implementation of the Agricultural Supply-Side Structural Reform}

Supply-side structural reform in rural areas is a major force for China's rural development. Currently, there is no problem in the quantity of grain production in China, however, the structural imbalance is serious, mainly manifested as the dislocation of the supply and demand structure of agricultural products, high production costs, excessive use of resources, etc., which has become a prominent shortcoming of China's economic and social development. In order to make up for this shortcoming and strengthen the advantages of China's agricultural development, China made comprehensive arrangements for optimizing the industrial structure of agricultural products, implementing green production mode, strengthening the drive of scientific and technological innovation, and strengthening rural reform at the beginning of 2017. This measure is of great significance for solving the problems of low land utilization rate in rural areas and imbalance of supply and demand structure of agricultural products, and it is also the core measure to promote the strategy of rural revitalization and the integrated development of urban and rural areas.

The promotion of integrated urban and rural development should be linked with the implementation of agricultural supply-side structural reform. With this as the main starting point, we shall further promote the reform of the quality and efficiency of the agricultural supply system. [8] On the premise of ensuring sufficient supply of agricultural products, varieties and quality can meet the demand of consumers to a certain extent, so that the effective supply of agricultural products can reach the level of reasonable structure and strong guarantee and promote the agricultural modernization. We will strengthen institutional supply, continue to improve the market-based allocation of property rights systems and elements, maximize the activation of themes, elements and markets, strengthen the systemic, holistic and synergistic nature of reform, deepen reform of the system of collective property rights in rural areas, adjust the pricing mechanism for agricultural products, abandon unreasonable restrictions on constrain farmers, strengthen farmers training, and fight against poverty.

\subsection{Blend into the Promotion of New Urbanization Construction}

The overall requirements of the Rural Revitalization Strategy are highly consistent with the basic characteristics of new-type urbanization. Coordinated development between urban and rural areas, ecological livability, effective governance, etc., and most importantly, human modernization. First of all, we need to re-shape the relationship between urban and rural areas, 
continue to replace agriculture with industry and supplement agriculture with industry, cities and towns drive the development of rural areas, with the focus on building rural public infrastructure, and gradually realizing a social public service system that covers all areas, benefits all and integrates urban and rural areas; actively guide urban talents, technology, capital and other elements to flow to rural areas to promote the integrated and interconnected development of industry, agriculture and service industry; secondly, we should realize ecological livability, adhere to green and sustainable development, and construct the spatial pattern, production mode and lifestyle of resource conservation and environmental protection; realize effective governance, further accelerate the establishment and improvement of the modern rural governance system, improve the self-control ability and awareness of farmers, seriously investigate and deal with corruption that infringes on the interests of farmers, and ensure the harmonious and orderly development of rural areas. [9]

\subsection{Support with the Realization of Agricultural Modernization}

Accelerating agricultural and rural modernization and realizing the organic connection between small farmers and modern development is one of the important contents of implementing the strategy of rural revitalization, which is also consistent with the previous goal of realizing agricultural modernization in China. [10] To promote the implementation of the Rural Revitalization Strategy, it is necessary to coordinate with the construction of modern agriculture deployment, and on this basis constantly improve. Promote agricultural modernization in depth, take agricultural mechanization as the prerequisite, scientific production technology as the driving force, agricultural industrialization as the important content, and agricultural informatization as the means to take the path of agricultural modernization that is characterized by efficient output, product safety, resource conservation and environmental friendliness; accelerate the development of modern agricultural production, operation and service system, improve the overall competitiveness of agriculture by using modern science and technology to serve agriculture and using modern modes of production to transform agriculture, foster new types of professional farmers and agricultural product operators, establish and improve the system of socialized agricultural services, and accelerate agricultural transformation and upgrading.

\subsection{Link with the Promotion of Rural Spiritual Civilization Construction}

Rural civilization is not only the fundamental task of the Construction of Rural Spiritual Civilization, but also a major task of the Rural Revitalization Strategy. The implementation of the Rural Revitalization Strategy should be coordinated with the construction of rural spiritual civilization. Energetically carry forward the excellent traditional Chinese culture, further promote the building of spiritual civilization in rural areas, transform social traditions, improve farmers' spiritual outlook and raise their spiritual and cultural level through village rules and regulations and other means; continue to increase funding, strengthen financial support, improve public culture in rural areas, and promote the coordinated development of medical, health, educational and service systems; make village affairs more open, improve the system of villagers' self-governance, recruit and utilize talented people, and give full play to their advantages in rural governance, strengthen legal system construction, raise farmers' awareness of legal rights protection, and while preserving rural culture, establish a rural governance system featuring trinity of rural autonomy, rule of law and rule of virtue to promote the urban-rural integrated development.

\section{Conclusion}

Socialism with Chinese characteristics has entered a new era, which indicates that China is transforming from being rich to strengthening, indicating that China is in a critical period of reform and a key node for the comprehensive construction of a socialist modernization power. [11] In the new era, China must always clearly understand the main contradictions in our society, highlight the problems of imbalance between urban and rural development, promote the integration of urban and rural development, improve the spiritual and cultural level of villagers, improve rural infrastructure construction, and build a new countryside with comprehensive politics, economy and cultural development.

\section{Acknowledgements}

This article is the research result of the Jiangsu Graduate Research and Innovation Program (No. KYCX18-2206)

\section{References}

[1] Chen Yanbing. Implementing the Strategy of Rural Revitalization, Promoting the Development of Urban and Rural Integration - Also on the Experience of Studying the Report of the 19th National Congress of the Communist Party of China [J]. China Economic and Trade Guide, 2017 (34), 52-55.

[2] Xi Jinping. Report on the 19th National Congress of the Communist Party of China [EB/OL]. Xinhuanet, 2017-10-18.

[3] Wang Xicheng. Promoting the Development of Urban and Rural Integration with the Srategy of Rural Revitalization [J]. Regional Economic Review, 2018, (03), 122-123.

[4] Liu Yansui. China's New Era Urban-rural Integration and Rural Revitalization [J]. Journal of Geographical Sciences, 2018, 73 (04), 637-650.

[5] Zhang Yanping. Reconstruction of China's Urban-rural Relationship Under the Rural Revitalization Strategy [J]. Agricultural Economy, 2018, (12), 68-70.

[6] Zhao Bingqin, Wu Lixia. The Integration of Urban and Rural Development is the Only Way to Revitalize the Countryside [J]. Journal of the Party School of Shijiazhuang Municipal Committee of the Communist Party of China, 2019, 21 (05), 36-39. 
[7] Wang Yiji, Wei Houkai. Rural Revitalization Strategy from the Perspective of Urban-Rural Integration Development: Proposing Background and Internal Logic [J]. Rural Economy, 2019, (01), 1-7.

[8] Chen Yuxin. Research on Rural Revitalization Strategy and Urban-Rural Integration Mechanism Innovation [J]. People's Forum - Academic Frontiers, 2018, (03), 72-76.

[9] Fu Cuilian. New Era to Promote Rural Revitalization Through Urban-rural Integration: Goals, Difficulties and Paths [J]. Journal of Tonghua Normal College, 2018, 39 (01), 1-8.

[10] Wang Yahua, Su Yiqing. Rural Revitalization-A New Strategy for China's Rural Development [J]. Journal of Central Socialist University, 2017, (06), 49-55.

[11] Tang Huimin. An Outline of the Three-Dimensional Dimension of Rural Revitalization Strategy [J]. Journal of Northwest Agricultural University of Science and Technology, 2019, 19 (03), 10-17

[12] Yang Yiqing. The Path Choice of Realizing Rural Revitalization in China from the Perspective of Urban and Rural Integration [J]. Modern Economics Discussion, 2018, (06), 101-106.

[13] Cheng Liangbo. International Experience and Reference of Rural Revitalization [J]. Village Science and Technology, 2018, (32), 30-32.

[14] He Wenhua. Rural Revitalization: The Logical Starting Point of the Integration of Urban and Rural Development in the New
Era $[J]$, Journal of Shanxi Agricultural University (Social Science Edition), 2019, 18 (01), 16-25.

[15] Lv Fengyong. The Fundamental Way of Rural Revitalization Strategy is to Integrate with Urban and Rural Areas [J]. China's national conditions, 2018, (06), 53-55.

[16] Wu Yuxing. The Great Significance and Realistic Path of Implementing the Strategy of Rural Revitalization-Interpretation from the Perspective of Urban-Rural Integration $[\mathrm{J}]$. China Agriculture Digest-Agricultural Engineering, 2019, 31 (03), 50-51.

\section{Biography}

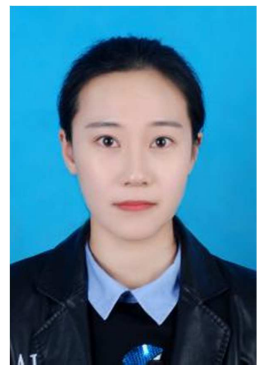

Ma Xiaoshuang (1993- ), female, Shangqiu, Henan, graduate student of Marxism college, Jiangsu University, mainly engaged in the study of Marxism in China. 